\title{
Green oxidation of alkenes in ionic liquid solvent by hydrogen peroxide over high performance Fe(III) Schiff base complexes immobilized on MCM-41
}

\author{
MOHAMMAD TAGHI GOLDANI ${ }^{\mathrm{a}, *}$, ALI MOHAMMADI ${ }^{\mathrm{b}, *}$ and REZA SANDAROOS ${ }^{\mathrm{c}}$ \\ a Department of Chemical Engineering, Birjand Branch, Islamic Azad University, Birjand 9717851367, Iran \\ ${ }^{\mathrm{b}}$ Department of Chemistry, Faculty of Sciences, Ferdowsi University of Mashhad, PO Box 91775, \\ Mashhad, Iran \\ ${ }^{c}$ Department of Chemistry, Faculty of Sciences, University of Birjand, Birjand, Iran \\ e-mail: mt.goldani@yahoo.com; a.mohammady62@yahoo.com
}

MS received 11 March 2012; revised 24 January 2014; accepted 27 January 2014

\begin{abstract}
A series of Fe(III) Schiff base complexes immobilized on MCM-41 were prepared and characterized by various physicochemical and spectroscopic methods. The complexes were used for oxidation of cyclohexene by $30 \%$ hydrogen peroxide in the presence and absence of ethylmethyl imidazolium chloride (EMIM) ionic liquid as solvent. The immobilized complexes proved to be effective catalysts and generally exhibited much higher catalytic performance than their homogeneous analogue. Catalytic performance of the complexes was also found to be closely related to the Schiff base ligands used. Additionally, ion liquid solvent efficiently improved all the catalytic performances. Finally, the reaction was extended to different alkenes using the heterogeneous complex $2-\mathrm{L}_{4}$. Among all the alkenes, those containing $\pi$-electron-withdrawing groups and trans-orientations exhibited lower tendency for oxidation.
\end{abstract}

Keywords. Immobilized complexes; alkene oxidation; hydrogen peroxide; ionic liquid; MCM-41.

\section{Introduction}

In the area of metal-catalysed oxidation, epoxidation of alkenes by heterogeneous and homogenous catalysts have attracted enormous attention, because expoxides are very precious intermediates in modern organic synthesis, and pharmacology and polymer industry. ${ }^{1-8}$ Among the efficient oxidants used commonly, molecular oxygen and hydrogen peroxide are economically and ecologically preferred. However, $\mathrm{H}_{2} \mathrm{O}_{2}$ is easier to handle, cheap and forms water as a byproduct. ${ }^{9}$ In comparison to supported catalysts, separation of catalyst from the reaction mixture is a typical disadvantage of homogenous catalysts. ${ }^{10,11}$ The supported metal complexes also show high catalytic activity $^{12,13}$ and selectivity ${ }^{14}$ in comparison to unsupported complexes. Unsupported metal complexes of Bdiketones ${ }^{15}$ and salen ${ }^{16}$ show low activity and selectivity in epoxidation of olefins in comparison to supported metal complexes. ${ }^{10,14,17-25}$ A popular approach for preparation of heterogeneous catalyst is to immobilize the homogeneous catalyst on the support through

\footnotetext{
*For correspondence
}

a surface-bound tether containing a functional group. This methodology has been employed successfully in the functionalization of various traditional types of silica supports. ${ }^{26-28}$ More recently, MCM-41 materials have been used extensively as ideal inorganic supports due to their high surface area and sharply distributed pore dimensions in the mesoporous ranges, which allow easy access of reactants to the active sites and also functionalization of bulky catalytic sites within the pores. ${ }^{29-31}$

It is well-known that the widespread use of traditional organic solvents in many chemical processes is an issue of great environmental concern. Hence, solventfree reactions and performing chemical reaction in ionic liquid solvents are topics of current interest in the context of environment-friendly chemical reactions. ${ }^{32}$

In the present investigation, a series of conventional Fe(III) Schiff base complexes were immobilized on MCM-41, and the resulting immobilized complexes were used for selective oxidation of alkenes by $30 \%$ hydrogen peroxide in the presence and absence of ethylmethyl imidazolium chloride (EMIM) ionic liquid as solvent. The role of Schiff base ligands and solvent on catalytic performances of the immobilized catalysts was also explored. 


\section{Experimental}

\subsection{General}

3-Aminopropyltriethoxysilane (APTES), salicylaldehyde, ethylenediamine, diethyltriamine, 1,2-diaminocyclohexane (chair conformation) and $o$-phenylenediamine supplied by Merck Chemical Co. (Darmstadt, Germany) and Fluka Co. and were used as received. Ligands and metal-complexes were characterized by spectroscopic measurements.

\subsection{Preparation of catalysts}

Synthetic procedure for pure siliceous MCM-41 (Si-MCM-41) is followed as described in literature. $^{33}$ 3-Aminopropyltriethoxysilane-modified MCM41 (APTES-MCM-41) was prepared according to a known procedure. ${ }^{34}$ Homogeneous Schiff base complexes were prepared by condensation of salicylaldehyde and appropriate diamines in 2:1 molar ratio. In a typical synthesis, diamine $(10 \mathrm{mmol})$ was suspended in absolute ethanol $(60 \mathrm{~mL})$. A solution of salicaldehyde $(20 \mathrm{mmol})$ in absolute ethanol $(60 \mathrm{~mL})$ was added dropwise with vigorous stirring of suspension. The resulting mixture was refluxed for $3 \mathrm{~h}$, then neutralized with the appropriate amount of $\mathrm{NaOH}$ to adjust the $\mathrm{pH}$ close to 7 , prior to the addition of solid $\mathrm{FeCl} 3$ (12 mmol). After refluxing for another $6 \mathrm{~h}$, the mixture was cooled and filtered under reduced pressure. The collected solid was washed with absolute ethanol and dried in air, then recrystallized from absolute ethanol. The homogeneous complexes were bound to MCM-41 by one axially coordinating propyl chain spacer, which allowed maximum conformational mobility of the active complexes. In order to prepare typical supported catalyst, a suspension of freshly dried APTESMCM-41 $(2 \mathrm{~g})$ in dry toluene $(50 \mathrm{~mL})$ was added to a solution of the prepared homogeneous complex $(8 \mathrm{mmol})$ in dry toluene $(50 \mathrm{~mL})$. The mixture was vigorously stirred under reflux for $6 \mathrm{~h}$. The resulting suspension was cooled and filtered through a fine-porous filter paper. The collected powder was washed overnight in a Soxhlet extractor using absolute ethanol and acetonitrile with an ethanol/acetonitrile molar ratio of 1:1 to remove free ligands and homogeneous complexes adsorbed on the external surface of support. The solid sample was further stirred in $0.01 \mathrm{~mol} / \mathrm{L} \mathrm{NaCl}$ solution for $24 \mathrm{~h}$ to remove the remaining uncoordinated metal ions. Finally, the mixture was filtered, and the obtained solid was dried in air at $80^{\circ} \mathrm{C}$ for $10 \mathrm{~h}$. By changing the diamine, a series of homogeneous Fe(III) Schiff base complexes (abbreviated as $1-\mathrm{L}_{1}-\mathrm{L}_{4}$ ) and their immobilized analogue (abbreviated as $2-\mathrm{L}_{1}-\mathrm{L}_{4}$ ) were prepared (figure 1).

\subsection{Characterization of catalysts}

Chemical analysis revealed that a small amount of carbon and nitrogen was present in the MCM-41 due to the remnant surfactant. Much increased carbon and nitrogen contents were observed in APTES-MCM-41, confirming its successful preparation. Elemental contents in all five homogeneous complexes were quite comparable with the calculated values, indicating that the as-prepared homogeneous complexes held the expected elemental composition (table 3). Diagnostic bands of FTIR spectra of the resulting immobilized complexes are listed in table 3. Successful preparation of homogeneous complexes could be confirmed by the presence of the characteristic bands at about $1600,600 \mathrm{~cm}^{-1}$, depending on the different ligands used, assigned to the $\mathrm{C}=\mathrm{N}$ stretching vibration of the imine groups. In addition, presence of two or three bands in the low frequency region $\left(450-550 \mathrm{~cm}^{-1}\right)$ indicated successful coordination of phenolic oxygen and azomethine nitrogen to the transition metal. In the FTIR spectra of immobilized complexes, apart from the bands in overlapping region of the silica backbone, the other bands of homogeneous complexes were all clearly observed though there were some marginal shifts in the positions of these bands due to immobilization. This indicates that quadridentate coordination structures of the homogeneous complexes survived the immobilization procedure.

The UV-vis spectra of the homogeneous complexes displayed one characteristic peak at about 390-450 nm typical of the metal-ligand band and one broad peak at about $570-610 \mathrm{~nm}$ associated with d-d transitions. The
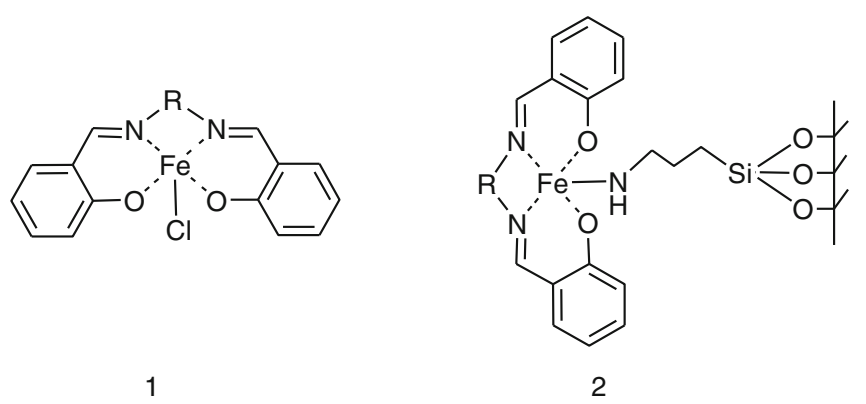

Figure 1. Homogeneous Fe(III) Schiff base complexes (1$\left.\mathrm{L}_{1}-\mathrm{L}_{4}\right)$ and their immobilized analogues $\left(2-\mathrm{L}_{1}-\mathrm{L}_{4}\right) . \mathrm{L}_{1}: \mathrm{R}=$ $-\mathrm{CH} 2 \mathrm{CH} 2-; \mathrm{L}_{2}: \mathrm{R}=-\mathrm{CH} 2 \mathrm{CH} 2 \mathrm{NHCH} 2 \mathrm{CH} 2-; \mathrm{L}_{3}: \mathrm{R}=\mathrm{C}_{\text {; }}$ $\mathrm{L}_{4}: \mathrm{R}=$ 牙. 
UV-vis spectra of all the immobilized complexes were quite similar to those of their corresponding homogeneous complexes, further confirming that the immobilized Fe(III) Schiff base complexes were successfully prepared.

\subsection{Catalytic performance}

A mixture of substrate and catalyst in the required molar ratio was stirred for about $10 \mathrm{~min}$ and then the corresponding amount of $\mathrm{H}_{2} \mathrm{O}_{2}$ was added. Required amount of solvent was also added when ionic liquid was used as solvent. After the mixture was heated to the desired temperature for the desired time, the products were filtered and purified by column chromatography using hexan/ethyl acetate 9:1 as eluent.

\section{Results and discussion}

Epoxidation of cyclohexene using homogeneous catalysts of $1-\mathrm{L}_{1}-\mathrm{L}_{4}$ and heterogeneous catalysts $2-\mathrm{L}_{1}-\mathrm{L}_{4}$ was selected as a template reaction (table 1). In order to compare catalytic performances (selectivity $\times$ conversion) which originate from reaction media, all the reactions were performed in the absence and presence of EMIM ionic liquid. When the reaction was performed over the immobilized complexes, on the whole, much higher catalytic performance was obtained than over their corresponding homogeneous complexes. Such obviously enhanced catalytic performance could, in part, be attributed to the ability of the support to highly disperse the active complexes and/or to control local concentrations of the reactant and product around active sites. The ligand structure was also, as usual, an important factor in determining the activity and selectivity of immobilized catalyst. In $2-\mathrm{L}_{1}$ and 2 $\mathrm{L}_{2}$, the bridge groups were all alkyl groups; however, $2-\mathrm{L}_{2}$ showed higher catalytic performance due to the additional $\mathrm{N}$ atom with isolated electron pair increasing donation capacity of the ligand. The bridge group in 2- $\mathrm{L}_{4}$ was phenyl which contributed to formation of the p-extended coordination structure. This could facilitate oxidation and so afford the best catalytic performance of $2-\mathrm{L}_{4}$. The worst catalytic performance, over $2-\mathrm{L}_{3}$, could be due to the higher stearic encumbrance around the Fe (III) centre, originating from the chair conformation of the bridge group. Consequently, catalytic performance observed over the immobilized complexes with different Schiff base ligands was in the following order: $2-\mathrm{L}_{4}>2-\mathrm{L}_{2}>2-\mathrm{L}_{1}>2-\mathrm{L}_{3}$. Supported catalysts exhibit better selectivity compared to their homogeneous congener. The epoxide ring-opening side reaction is catalysed mainly by the acidic metal sites. Probably, changes in the surface properties produced by electron donation of support decrease acidity of the surface, preventing epoxides from converting to diols.

Totally, all the catalysts showed much higher catalytic performance when ionic liquid was used as solvent (table 1).

The reaction was extended to different alkenes using catalyst $2-\mathrm{L}_{4}$ in ionic liquid solvent from which good to excellent catalytic performances were obtained (tables 2 and 3 ).

Conjugated $\pi$-electron-withdrawing groups with double bond of alkenes decrease reactivity for epoxidation (compare entries 1 and 5). Although, the oxygen atom is an $\sigma$-acceptor, $\pi$-resonance will increase electron density on reaction centre through the oxygen lone pairs. Accordingly, presence of a methoxy group in methyl cinnamate (entry 6) probably causes enhanced reactivity of this alkene with respect to

Table 1. Epoxidation of cyclohexene with $\mathrm{H}_{2} \mathrm{O}_{2}$ in the presence of supported and unsupported Fe (salen) complexes. ${ }^{\text {a }}$

\begin{tabular}{|c|c|c|c|c|c|c|c|}
\hline Entry & Complex & $\begin{array}{c}\text { Cyclohexene } \\
\text { conversion }^{\mathbf{b}}(\%)\end{array}$ & $\begin{array}{l}\text { Selectivity } \\
\text { epoxide }(\%)\end{array}$ & Entry & Complex & $\begin{array}{c}\text { Cyclohexene } \\
\text { conversion }^{\mathbf{b}}(\%)\end{array}$ & $\begin{array}{l}\text { Selectivity } \\
\text { epoxide }(\%)\end{array}$ \\
\hline 1 & $1-\mathrm{L}_{1}$ & 38 & 66 & 9 & $2-\mathrm{L}_{1}$ & 73 & 96 \\
\hline $2^{d}$ & $1-\mathrm{L}_{1}$ & 55 & 75 & $10^{\mathrm{d}}$ & $2-\mathrm{L}_{1}$ & 88 & 100 \\
\hline 3 & $1-\mathrm{L}_{2}$ & 44 & 72 & 11 & $2-L_{2}$ & 74 & 100 \\
\hline $4^{d}$ & $1-\mathrm{L}_{2}$ & 60 & 82 & $12^{\mathrm{d}}$ & $2-\mathrm{L}_{2}$ & 91 & 100 \\
\hline 5 & $1-\mathrm{L}_{3}$ & 33 & 61 & 13 & $2-\mathrm{L}_{3}$ & 43 & 65 \\
\hline $6^{\mathrm{d}}$ & $1-\mathrm{L}_{3}$ & 51 & 73 & $14^{\mathrm{d}}$ & $2-\mathrm{L}_{3}$ & 57 & 75 \\
\hline 7 & $1-\mathrm{L}_{4}$ & 53 & 77 & 15 & $2-\mathrm{L}_{4}$ & 78 & 100 \\
\hline $8^{d}$ & $1-\mathrm{L}_{4}$ & 69 & 90 & $16^{\mathrm{d}}$ & $2-\mathrm{L}_{4}$ & 95 & 100 \\
\hline
\end{tabular}

${ }^{\mathrm{a}}$ Molar ratios for Fe: cyclohexene: oxidant $=0.02: 1: 2$, Time $=20 \mathrm{~min}$, Temperature $=50^{\circ} \mathrm{C}$

${ }^{\mathrm{b}}$ Conversion $=(\mathrm{mol}$ of cyclohexene reacted $/ \mathrm{mol}$ of cyclohexene in the feed $) \times 100$

${ }^{\mathrm{c}}$ Selectivity $=(\mathrm{mol}$ of cyclohexene converted to the epoxide $/ \mathrm{mol}$ of cyclohexene reacted $) \times 100$

${ }^{\mathrm{d}}$ Ionic liquid was used as solvent $(3 \mathrm{~mL})$ 
Table 2. Epoxidation of alkenes with $\mathrm{H}_{2} \mathrm{O}_{2}$ in the presence of supported complex 2- $\mathrm{L}_{4}^{\mathrm{a}}$.

$\begin{gathered}\text { Alkene } \\ \text { conversion } \mathbf{b}^{\mathbf{b}}(\%)\end{gathered}$
Selectivity(\%)

${ }^{a}$ Molar ratios for Fe: substrate: oxidant $=0.02: 1: 2$, Time $=20$ min, Temperature $=50^{\circ} \mathrm{C}$, ionic liquid $=3 \mathrm{~mL}$

${ }^{\mathrm{b}}$ Conversion $=(\mathrm{mol}$ of alkene reacted $/ \mathrm{mol}$ of alkene in the feed $) \times 100$

${ }^{\mathrm{c}}$ Selectivity $=(\mathrm{mol}$ of alkene converted to the products $/ \mathrm{mol}$ of alkene reacted $) \times 100$

${ }^{\mathrm{d}-\mathrm{f}}$ Reused complex $2-\mathrm{L}_{4}$

Table 3. Elemental compositions and spectroscopic data for the complex samples. ${ }^{1}$

\begin{tabular}{lllll}
\hline & \multicolumn{2}{l}{ Found (calcd.) $\%$} & \multicolumn{2}{l}{ Spectroscopic data } \\
\cline { 2 - 5 } Samples & $\mathrm{C}$ & $\mathrm{N}$ & FTIR $\left(\mathrm{cm}^{-\mathbf{1}}\right)$ & $\lambda_{\max }(\mathrm{nm})$ \\
\hline MCM - 41 & 2.0 & 0.1 & - & - \\
APTES- MCM -41 & 5.9 & 2.3 & - & - \\
1-L1 & $53.68(53.74)$ & $7.80(7.83)$ & $1615,585,465$ & 390,600 \\
1-L2 & $53.88(53.96)$ & $10.46(10.49)$ & $1620,612,522$ & 390,609 \\
1-L3 & $58.30(58.35)$ & $6.75(6.80)$ & $1620,602,535$ & 416,606 \\
1-L4 & $59.17(59.22)$ & $6.86(6.91)$ & $1619,539,462$ & 446,575 \\
2-L1 & 2.6 & 1.5 & $1612,585,465$ & 390,600 \\
2-L2 & 1.9 & 1.8 & $1608,612,522$ & 390,610 \\
2-L3 & 3.1 & 1.4 & $1611,602,535$ & 417,606 \\
2-L4 & 3.0 & 1.5 & 1610 & 447,578 \\
\hline
\end{tabular}

${ }^{1}$ FTIR spectra of all samples were recorded in $\mathrm{KBr}$ disks at room temperature on a Shimadzu (model 8201 PC) spectrophotometer. UV-vis spectra were recorded on a Shimadzu (model 2501 PC) 
benzylideneacetone (entry 7). Decreased reactivity of 2,4,4-trimethyl-1-pentene (entry 9) seems to be due to the stearic bulk of tert-butyl substituents of the alkene. Comparison of entries 9 and 10 may indicate dominance of stearic factors in epoxidation. More reactivity of cis-alkenes in comparison to trans-ones may be due to the cis-orientation of the substituents which facilitate the approach of the alkene to the active oxidant (compare entries 7 with 8 and 13 with 14). In general, since alkenes act as nucleophiles attacking the peroxide (or the active oxidant) bond, the rate of epoxidation is enhanced by increasing alkyl substitutions (compare entries 7 and 11). It should be noted that the base-catalysed epoxidation of alkenes with $\mathrm{H}_{2} \mathrm{O}_{2}$ prefers electron-deficient alkenes, i.e., $\alpha, \beta$-unsaturated carbonyl compounds, to the electron-rich ones, because the rate determining step seems to be the nucleophilic addition of $\mathrm{HO}^{-} \mathrm{O}^{-}$to the $\mathrm{C}, \mathrm{C}$ double bond. ${ }^{7}$ We next examined reusability of catalyst $2-\mathrm{L}_{4}$ for epoxidation of cyclohexene. The obtained results revealed no negligible activity loss over consecutive recycling (table 2 , entries 2-4).

\section{Conclusion}

A series of immobilized Fe(III) Schiff base complexes were successfully prepared and they exhibited promising catalytic performance toward selective oxidation of cyclohexene by $30 \% \mathrm{H}_{2} \mathrm{O}_{2}$. Significant differences in catalytic performance of the immobilized complexes could be attributed to their different ligand structures, and the immobilized complex $2-\mathrm{L}_{4}$ with $\pi$ extended coordination structure was found to exhibit the best catalytic performance. Among the alkenes, those containing $\pi$-electron-withdrawing groups and trans-orientations exhibited lower reactivity. Additionally, ionic liquid solvent efficiently improved all the catalytic performances.

\section{References}

1. Hauser A, Cokoja M and Kühn E 2013 Catal. Sci. Technol. 3552

2. Sherry B D and Fürstner A 2008 Acc. Chem. Res. 41 1500

3. Saisaha P, de Boer J W and Browne W R 2013 Chem. Soc. Rev. 42 2059-2074

4. Visuvamithiran $\mathrm{P}$, Palanichamy $\mathrm{M}$, Shanthi $\mathrm{K}$ and Murugesan V 2013 Appl. Catal. A: Gen. 462 31-38
5. Hashmi A S K, Graf K, Ackermann M and Rominger F 2013 Chem. Catal. Chem. 5 1092-1095

6. Gaspar H, Andrade M, Pereira C, Pereira A M, Rebelo S L H, Araújo J P, Pires J, Arvalho A P and Freire C 2013 Catal. Today 203 103-110

7. Prat I, Font D, Company A, Junge K, Ribas X, Beller M and Costas M 2013 Adv. Synth. Catal. 355 947-956

8. Backvall J E 2004 Modern oxidation methods (Weinheim: Wiley-VCH)

9. Grigoropoulou G, Clark J H and Elings J A 2003 Green Chem. 5 1-7

10. Andrea B, Martino S and Beniditto C 2006 J. Mol. Catal. A: Chem. 2501

11. Shahram T, Hossein H M, Valiollah M, Mazid M and Gholamhossein G 2006 Inorg. Chem. 9575

12. Yoo D W, Han J H, Nam S H, Kim C and Lee J K 2006 Inorg. Chem. Commun. 9654

13. Akelah A, Rehab A, Kenawy E R and Zeid A M S 2006 J. Appl. Polym. Sci. 1011121

14. Stefan B, Agniesszker S and Beata S 2006 Appl. Catal. A: Gen. 30985

15. Blaz E and Pielichowski J 2006 Molecules 11115

16. Lutsa T, Supruna W, Hofmannb D, Klepela O and Pap H 2007 J. Mol. Catal. A: Chem. 26116

17. Zsigmond A, Boger K and Notheisz F 2003 J. Catal. 213103

18. Yulong Q and Daodao H 2005 Gaofenzi Tongbao 131

19. Shahram $\mathrm{T}$, Hossein $\mathrm{H} \mathrm{M}$, Moghadam M V and Gholamhossein M G 2006 J. Mol. Catal. A: Chem. 255249

20. Beloglazkina E K, Majangu A G, Romashkima R B and Zyk N V 2006 Tetrahedron Lett. 472957

21. Kamata K, Yonehara K, Sumida Y, Hirata K, Nojima S and Mizuno N 2011 Angew. Chem. Int. Ed. 5012062

22. Ahn J H, Kim J C, Ihm S K, Oh C G and Sherrington D C 2005 Ind. Eng. Chem. Res. 448560

23. Wang Q, Wang L, Chen J, Wu Y and Mi Z 2007 J. Mol. Catal. A: Chem. 27373

24. Ziolek M 2004 Catal. Today 90145

25. Olason G and Sherrington D C 1999 React. Funct. Polym. 42163

26. Zhou X G, Yu X Q, Huang J S, Li S G, Li L S and Che C M 1999 Chem. Commun. 1789

27. Zhang H, Xiang S and Li C 2005 Chem. Commun. 1209

28. Xiang S, Zhang Y, Xin Q and Li C 2002 Chem. Commun. 2696

29. Oliveira P, Machado A, Ramos A M, Fonseca I, Braz Fernandes F M, Botelho do Rego A M and Vital J 2009 Micropor. Mesopor. Mat. 120432

30. Demicheli G, Maggi R, Mazzacani A, Righi P, Sartori $\mathrm{G}$ and Bigi F 2001 Tetrahedron Lett. 422401

31. Singh U G, Williams R T, Hallam K R and Allen G C 2005 J. Solid State Chem. 1783405

32. Formentin P, Garcia H and Leyva A 2004 J. Mol. Catal. A: Chem. 214137

33. Kresge C T, Leonowicz M E, Roth W J, Vartuli J C and Beck J S 1992 Nature 359710

34. Baleiza C, Gigante B, Sabater M J, Garcia H and Corma A 2002 Appl. Catal A: Gen. 228279 